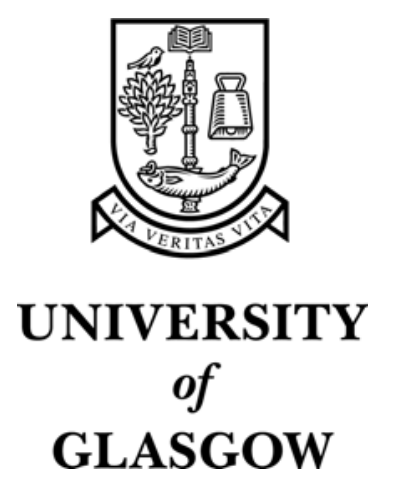

Laing, E W and Diver, D A (2005) On damped Bernstein modes in a weakly relativistic pair plasma. Physical Review E 72(3):036409.

http://eprints.gla.ac.uk/3154/ 


\title{
On damped Bernstein modes in a weakly relativistic pair plasma
}

\author{
E W Laing and D A Diver \\ Dept of Physics and Astronomy, Kelvin Building, \\ University of Glasgow, Glasgow G12 8QQ, Scotland UK.*
}

(Dated: July 12, 2005)

\begin{abstract}
Relativistic Bernstein modes are not totally undamped, but have a small, negative definite imaginary frequency component which peaks where the frequency is closest to the rest cyclotron harmonic.
\end{abstract}

PACS numbers: 52.25.Dg, 52.25.Xz, 52.27.Ep, 52.27.Ny, 52.35.Fp

\section{INTRODUCTION}

In our previous paper on Bernstein modes in a weakly relativistic treatment based on Maxwellian distributions [1] we claimed that contributions due to the singularities which occurred at the resonances $\gamma^{2}=n^{2} / \hat{\omega}^{2}$ exactly cancelled, leaving the modes undamped. However it has since been pointed out [2] that the path of integration proposed in [1] to avoid the singularities was incorrect. The correct path should have been below the singularity on the negative $\hat{p}_{\|}$-axis, and above it on the positive $\hat{p}_{\|^{-}}$ axis. As a result, the contributions add, and the modes are damped.

In this brief article we calculate the damping of the relativistic Bernstein modes, assuming that the damping is weak. More precisely, given that the frequency of a Bernstein wave of normalised wave-number $\kappa=$ $(2 / a)^{1 / 2} k_{\perp} c / \Omega_{0}$ is $\hat{\omega}=\omega / \Omega_{0}$, we now write $\hat{\omega}=\hat{\omega}_{r}+i \hat{\omega}_{i}$, and assume that $\left|\hat{\omega}_{i} / \hat{\omega}_{r}\right| \ll 1$. We will use the same notation throughout as in [1], so that $a=m_{e} c^{2} /\left(k_{B} T\right)$ is the relativistic parameter, and $\Omega_{0}=e B_{0} / m_{e}$ is the rest cyclotron frequency for an electron or positron.

The full dispersion relation obtained in [1] is

$$
\begin{aligned}
& \hat{\omega}^{2}-2 \hat{\omega}_{p}^{2}= \\
& \frac{4 \hat{\omega}_{p}^{2} a^{5 / 2}}{\sqrt{2 \pi} \hat{\omega}^{2} \hat{k}_{\perp}^{2}} \sum_{n=1}^{\infty} n^{4} \int_{0}^{\infty} \mathrm{d} \hat{p}_{\perp} \hat{p}_{\perp} J_{n}^{2}\left(\hat{k}_{\perp} \hat{p}_{\perp}\right) e^{-a \hat{p}_{\perp}^{2} / 2} \\
& \times \int_{-\infty}^{\infty} \mathrm{d} \hat{p}_{\|} \frac{e^{-a \hat{p}_{\|}^{2} / 2}}{1+\hat{p}_{\|}^{2}+\hat{p}_{\perp}^{2}-n^{2} / \hat{\omega}^{2}}
\end{aligned}
$$

where $\hat{\omega}_{p}=\omega_{p} / \Omega_{0}$ is the normalised plasma frequency, $\hat{p}_{\|}=p_{\|} /\left(m_{e} c\right)$ and $\hat{p}_{\perp}=p_{\perp} /\left(m_{e} c\right)$.

Consider the properties of the second integral in Eq. (1). In the limit $\left|\hat{\omega}_{i} / \hat{\omega}_{r}\right| \ll 1$,

$$
\frac{n^{2}}{\hat{\omega}^{2}} \approx \frac{n^{2}}{\hat{\omega}_{r}^{2}}\left(1-2 i \frac{\hat{\omega}_{i}}{\hat{\omega}_{r}}\right)
$$

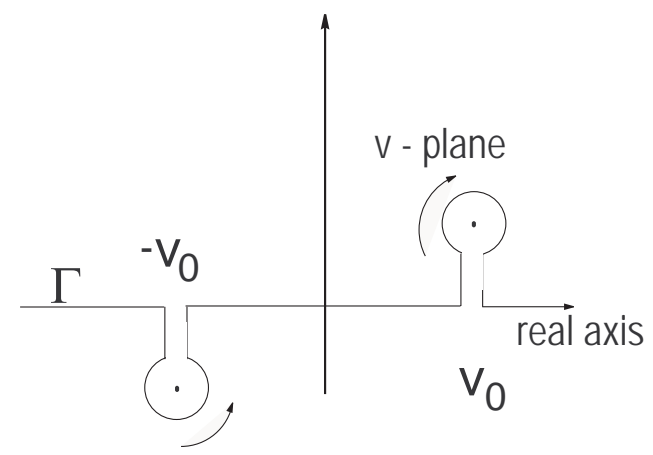

FIG. 1: Definition of the contour for the integral in Eq. (9).

Hence the integral becomes

$$
I=\int_{-\infty}^{\infty} \mathrm{d} \hat{p}_{\|} \frac{e^{-a \hat{p}_{\|}^{2} / 2}}{1+\hat{p}_{\|}^{2}+\hat{p}_{\perp}^{2}-n^{2} / \hat{\omega}_{r}^{2}+2 i n^{2} \hat{\omega}_{i} / \hat{\omega}_{r}^{3}}
$$

For convenience, define

$$
b_{n}^{2}=n^{2} / \hat{\omega}_{r}^{2}-1
$$

so that it is apparent that a different treatment of the integral $I$ is required depending on the sign of $b_{n}^{2}$, and the relative sizes of $b_{n}^{2}$ and $\hat{p}_{\perp}^{2}$.

We will use throughout the notation

$$
\begin{aligned}
x_{n}^{2} & =a b_{n}^{2} / 2 \\
y_{n} & =a n^{2} \hat{\omega}_{i} / \hat{\omega}_{r}^{3}<0 \\
v^{2} & =a \hat{p}_{\|}^{2} / 2 \\
x^{2} & =x_{n}^{2}-a \hat{p}_{\perp}^{2} / 2
\end{aligned}
$$

Case (i) $b_{n}^{2}>0, \quad b_{n}^{2}>\hat{p}_{\perp}^{2}$

The integral terms in Eq. (1) can be written in the simpler form

$$
\begin{gathered}
I=\sqrt{2 / a} \int_{0}^{x_{n}} x e^{-x_{n}^{2}+x^{2}} J_{n}^{2}\left(\kappa \sqrt{x_{n}^{2}-x^{2}}\right) \mathrm{d} x \\
\times \int_{\Gamma} \frac{e^{-v^{2}} \mathrm{~d} v}{v^{2}-x^{2}+i y_{n}}
\end{gathered}
$$


in which $\Gamma$ is the Landau contour given in Fig. 1. The poles are at $\pm v_{0}$, where $v_{0}=\sqrt{x^{2}-i y_{n}}$.

For the singular integral in (9), the contribution from the poles is given by

$$
-2 \pi i \frac{e^{-x^{2}+i y_{n}}}{v_{0}}
$$

using the Residue theorem. The integration along the real line then yields

$$
\int_{-\infty}^{\infty} \frac{\left(v^{2}-x^{2}\right) e^{-v^{2}} \mathrm{~d} v}{\left(v^{2}-x^{2}\right)^{2}+y_{n}^{2}}-i y_{n} \int_{-\infty}^{\infty} \frac{e^{-v^{2}} \mathrm{~d} v}{\left(v^{2}-x^{2}\right)^{2}+y_{n}^{2}}
$$

with all other contributions cancelling. Note that the integrals above are both real.

Case (ii) $b_{n}^{2}>0, \quad b_{n}^{2}<\hat{p}_{\perp}^{2}$

Here the $\hat{p}_{\|}$integral takes the form

$$
\int_{-\infty}^{\infty} \frac{e^{-v^{2}} \mathrm{~d} v}{v^{2}+x^{2}+i y_{n}}
$$

and is non-singular. This integral can be expressed in terms of real and imaginary parts as

$$
\int_{-\infty}^{\infty} \frac{e^{-v^{2}}\left(v^{2}+x^{2}\right)}{\left(v^{2}+x^{2}\right)^{2}+y_{n}^{2}} \mathrm{~d} v-i y_{n} \int_{-\infty}^{\infty} \frac{e^{-v^{2}}}{\left(v^{2}+x^{2}\right)^{2}+y_{n}^{2}} \mathrm{~d} v
$$

When $y_{n}=0$, the second integral vanishes, as expected.

\section{Case (iii) $n<\hat{\omega}_{r}$}

This is similar to case (ii), but now $b_{n}^{2}<0$; the $\hat{p}_{\perp}$ integral is as before, subject to this proviso.

The dispersion relation for frequency $m-1<\hat{\omega}_{r}<m$ where $m \geq 2$ is an integer and $C=8 \sqrt{\pi} \hat{\omega}_{p}^{2} a / \kappa^{2}$ can be written

$$
\begin{aligned}
& \hat{\omega}_{r}^{2}+2 i \hat{\omega}_{i} \hat{\omega}_{r}-2 \hat{\omega}_{p}^{2}=\frac{C}{\hat{\omega}_{r}^{2}}\left(1-2 i \frac{\hat{\omega}_{i}}{\hat{\omega}_{r}}\right) \times \\
& {\left[\sum_{n=1}^{m-1} n^{4} A_{n}^{\mathrm{III}}+\sum_{n=m}^{\infty} n^{4}\left(A_{n}^{\mathrm{I}}+A_{n}^{\mathrm{II}}-i B_{n}\right)\right]}
\end{aligned}
$$

in which

$$
\begin{gathered}
A_{n}^{\mathrm{I}}=e^{-x_{n}^{2}} \int_{0}^{x_{n}} x e^{x^{2}} J_{n}^{2}\left(\kappa \sqrt{x_{n}^{2}-x^{2}}\right) \times \\
{\left[q_{1}\left(x, y_{n}\right)-i y_{n} q_{2}\left(x, y_{n}\right)\right] \mathrm{d} x} \\
A_{n}^{\mathrm{II}}=e^{-x_{n}^{2}} \int_{0}^{\infty} x e^{-x^{2}} J_{n}^{2}\left(\kappa \sqrt{x_{n}^{2}+x^{2}}\right) \times \\
A_{n}^{\mathrm{III}}=e^{-x_{n}^{2}} \int_{\left.\mid p_{1}\left(x, y_{n}\right)-i y_{n} p_{2}\left(x, y_{n}\right)\right] \mathrm{d} x}^{\infty} x e^{-x^{2}} J_{n}^{2}\left(\kappa \sqrt{x^{2}+x_{n}^{2}}\right) \times \\
B_{n}=2 e^{-x_{n}^{2}} \int_{0}^{x_{n}} \frac{x J_{n}^{2}\left(\kappa \sqrt{x_{n}^{2}-x^{2}}\right)}{\sqrt{x^{2}-i y_{n}}} e^{i y_{n}} \mathrm{~d} x
\end{gathered}
$$

and where the following additional function definitions have been used:

$$
\begin{aligned}
& p_{1}(x, y)=\frac{1}{\pi} \int_{-\infty}^{\infty} \mathrm{d} v \frac{e^{-v^{2}}\left(v^{2}+x^{2}\right)}{\left(v^{2}+x^{2}\right)^{2}+y^{2}} \\
& p_{2}(x, y)=\frac{1}{\pi} \int_{-\infty}^{\infty} \mathrm{d} v \frac{e^{-v^{2}}}{\left(v^{2}+x^{2}\right)^{2}+y^{2}} \\
& q_{1}(x, y)=\frac{1}{\pi} \int_{-\infty}^{\infty} \mathrm{d} v \frac{e^{-v^{2}}\left(v^{2}-x^{2}\right)}{\left(v^{2}-x^{2}\right)^{2}+y^{2}} \\
& q_{2}(x, y)=\frac{1}{\pi} \int_{-\infty}^{\infty} \mathrm{d} v \frac{e^{-v^{2}}}{\left(v^{2}-x^{2}\right)^{2}+y^{2}}
\end{aligned}
$$

Note that in the limit $y_{n} \rightarrow 0, p_{1} \rightarrow e^{x^{2}} \operatorname{erfc}(x), q_{1} \rightarrow$ $i e^{-x^{2}} \operatorname{erf}(i x)$ and $A_{n}^{\mathrm{II}}$ and $A_{n}^{\mathrm{III}}$ become purely real; these then agree with the definitions given in Eqs. (48-49) of [1]. The real part of $A_{n}^{\mathrm{I}}$ similarly agrees with Eq. (47) of [1], but there is an imaginary contribution from the changed contour.

The procedure now, as discussed earlier, is to extract the real and imaginary parts of the dispersion relation, and to make the assumption that the real part is relatively unchanged from our earlier paper [1]. In this way, we can use the imaginary part of the dispersion relation to define an implicit relationship that will yield $\hat{\omega}_{i}$ as a function of $\hat{\omega}_{r}$ and the plasma parameters. It will turn out that we must retain the effects of $y_{n}$ beyond the linear approximation in order to avoid singular integrals. This is not the case when considering the real part, hence justifying the approximation of setting $\hat{\omega}_{i}=0$ there.

In order to separate the real and imaginary parts of the dispersion relation, we introduce the following notation 


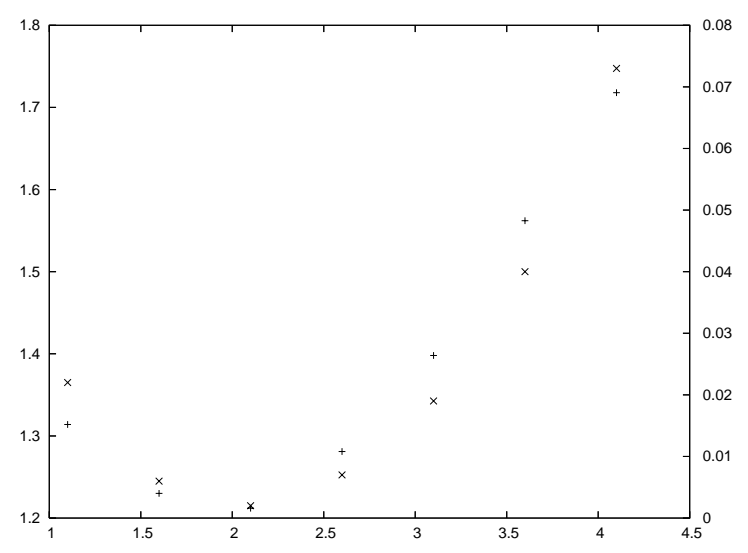

FIG. 3: Caption as for Fig. 2, showing the real and imaginary parts on the lower loop of the same dispersion curve.

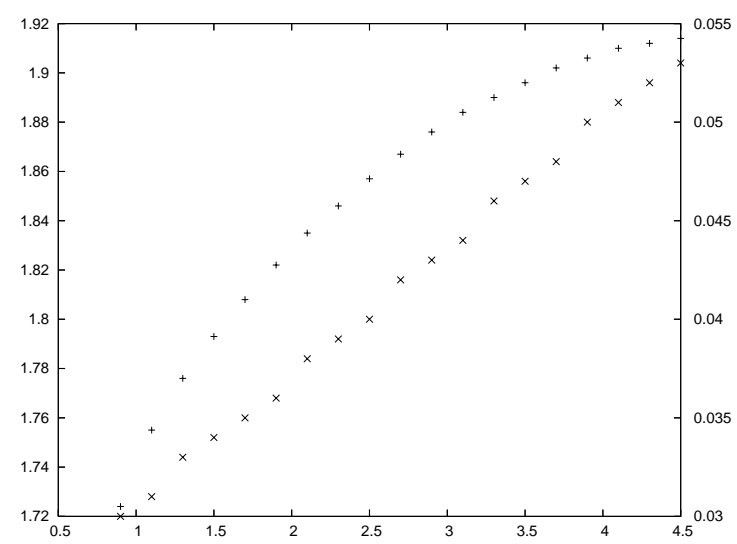

FIG. 4: Pluses $(+)$ show the upper loop of the dispersion curve $\hat{\omega}_{r}$ (left y-axis) versus $\kappa$ (x-axis) for $a=20$, and $1<$ $\hat{\omega}_{r}<2$. The crosses $(\times)$ show how $-\hat{\omega}_{i}$ (right $\mathrm{y}$-axis) varies as a function of $\kappa$ along the dispersion curve.

\section{SUMMARY}

This short article updates our original calculations [1] by showing that Bernstein modes in relativistic pair plasmas are indeed damped, albeit very weakly. The classical electron-ion picture suggests that this damping should occur close to the classical cyclotron resonances at $\omega \approx n \omega_{c}$ [3]. Our computed damping terms are largest closest to the cyclotron harmonics on the upper loop of each dispersion curve; $\hat{\omega}_{i}$ is comparatively smaller on the lower branch mainly because $x_{m}$ is larger in that lower frequency part of the curve, and so $A_{m 2}^{\mathrm{I}}$ is more dominant than $B_{m 1}$, leading to a smaller $\hat{\omega}_{i}$. This can readily be seen from the simple approximation given in Eq. (36).

Note also that we have shown in earlier work [1] that relativistic effects significantly change the shape and location of the dispersion curves for pair-plasma Bernstein modes such that they do not remain localised near a particular harmonic. In fact, most of the Bernstein mode dispersion curves are free from significant damping, and therefore the associated waves propagate largely without

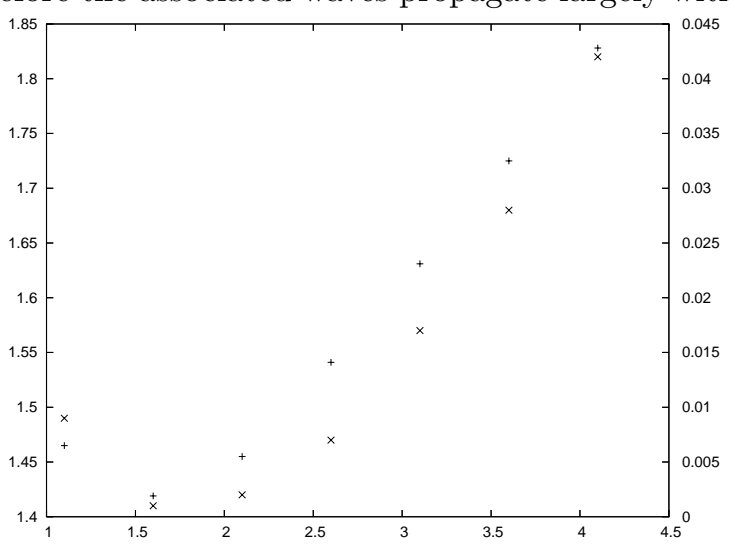

FIG. 5: Caption as for Fig. 4, showing the real and imaginary parts on the lower loop of the same dispersion curve.

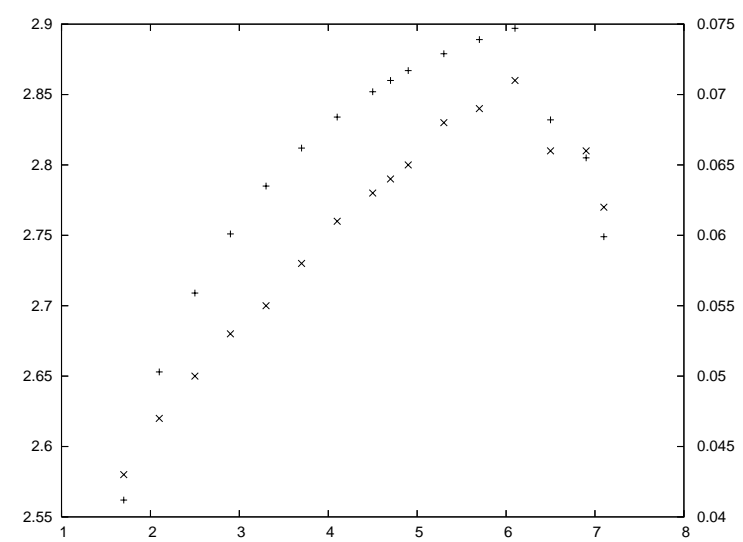

FIG. 6: Pluses (+) show the upper loop of the dispersion curve $\hat{\omega}_{r}$ (left y-axis) versus $\kappa$ (x-axis) for $a=20$, and $2<$ $\hat{\omega}_{r}<3$. The crosses $(\times)$ show how $-\hat{\omega}_{i}$ (right $\mathrm{y}$-axis) varies as a function of $\kappa$ along the dispersion curve.

hindrance.

\section{AKNOWLEDGMENTS}

It is a pleasure to acknowledge valuable and constructive comment from R A Cairns and C N LashmoreDavies. We are grateful to PPARC for partial funding of this research.
[1] D. A. Keston, E. W. Laing and D. A. Diver, Phys. Rev. E 67, 036403 (2003)
[2] R. A. Cairns and C. N. Lashmore-Davies, private communication 


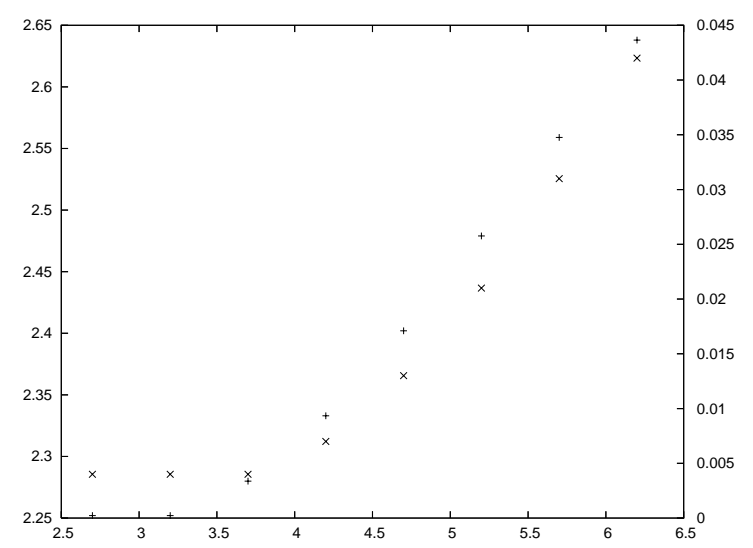

[3] R. A. Cairns and C. N. Lashmore-Davies, p.430, CP595 Radio Frequency Power in Plasmas: $14^{1} 4$ Topical Conf., T. K. Mau, J. deGrassie, editors, AIP 0-7354-0038-5 (2001)

FIG. 7: Caption as for Fig. 6, showing the real and imaginary parts on the lower loop of the same dispersion curve. 